Research Article

\title{
Some Properties of Curvature Tensors and Foliations of Locally Conformal Almost Kähler Manifolds
}

\author{
Ntokozo Sibonelo Khuzwayo (iD) and Fortuné Massamba (iD) \\ School of Mathematics, Statistics and Computer Science, University of Kwazulu-Natal, Private Bag X01, \\ Scottsville 3209, South Africa \\ Correspondence should be addressed to Fortuné Massamba; massfort@yahoo.fr
}

Received 23 October 2020; Revised 10 December 2020; Accepted 13 December 2020; Published 18 February 2021

Academic Editor: Ildefonso Castro

Copyright (C) 2021 Ntokozo Sibonelo Khuzwayo and Fortuné Massamba. This is an open access article distributed under the Creative Commons Attribution License, which permits unrestricted use, distribution, and reproduction in any medium, provided the original work is properly cited.

\begin{abstract}
We investigate a class of locally conformal almost Kähler structures and prove that, under some conditions, this class is a subclass of almost Kähler structures. We show that a locally conformal almost Kähler manifold admits a canonical foliation whose leaves are hypersurfaces with the mean curvature vector field proportional to the Lee vector field. The geodesibility of the leaves is also characterized, and their minimality coincides with the incompressibility of the Lee vector field along the leaves.
\end{abstract}

\section{Introduction}

The study of manifolds whose metric is locally conformal to an almost Kähler metric is considered as one of the most interesting studies in the field of differential geometry (see [1] for details and references therein). This is because of its richness in the theory that is applicable in physics, algebraic geometry, symplectic geometry, etc. To our knowledge, locally conformal (almost) Kähler structures were first studied by Libermann [2] in the 1950s. In 1966, Gray [3] also contributed to the study by considering (almost) Hermitian manifolds whose metric is conformal to a local (almost) Kähler metric. However, globally conformal (almost) Kähler manifolds share the same topological properties with locally conformal (almost) Kähler manifolds [4]. It is therefore provocative to consider those almost Hermitian manifolds whose metric is locally conformal to an almost Kähler metric. The difference between locally conformal Kähler manifolds and locally conformal almost Kähler manifolds is the condition of integrability of an almost complex structure. This is equivalent to an almost complex structure being parallel with respect to a globally defined connection or the vanishing of a Nijenhuis tensor. Therefore, the geometric properties which do not depend on the almost complex structure will apply to both of these manifolds.

Libermann defined a locally conformal (almost) Kähler metric as a metric $g$ at which in the neighborhood of each point of an almost Hermitian manifold, it is locally conformal to an (almost) Kähler metric.

In this paper, we investigate some properties of curvature tensors and foliations of locally conformal almost Kähler manifolds. For the foliations, we pay attention to the ones that arise naturally when the Lee form is nowhere vanishing. The paper is organized as follows. In Section 2, we recall the definition of locally conformal almost Kähler structures supported by an example. In Section 3, we deal with curvature tensors. The latter generalizes those found by Olszak in [5]. Under some conditions, we prove that a class of locally conformal almost Kähler structures is a subclass of almost Kähler structures. Section 4 is devoted to the canonical foliations that arise for the nonvanishing Lee form. We prove that these foliations are Riemannian if and only if the Lee vector field is autoparallel. We also prove that the locally conformal almost Kähler manifolds contain leaves with mean curvature vector field proportional to the Lee vector field, and their geodesibility coincides with the killing 
condition of the Lee vector field. The latter is incompressible along the leaves if and only if the leaves are minimal.

\section{Locally Conformal Almost Kähler Metrics}

Let $M$ be a $2 n$-dimensional almost Hermitian manifold with the metric $g$ and the almost complex structure $J$ satisfying

$$
\begin{aligned}
J^{2} & =-\llbracket, \\
g(J X, J Y) & =g(X, Y),
\end{aligned}
$$

for any vector fields $X$ and $Y$ tangent to $M$, where $\llbracket$ stands for the identity transformation of tangent bundle TM. Then, for any vector fields $X$ and $Y$, the tensor

$$
\Omega(X, Y)=g(X, J Y)
$$

defines the fundamental 2-form of $M$ which is nondegenerate and gives an almost symplectic structure on $M$. If $\Omega$ is closed, i.e., $d \Omega=0$, then $(M, J, g)$ is called an almost Kähler manifold [6].

Now, let $(M, J, g)$ be a $2 n$-dimensional almost Hermitian manifold. Such a manifold is said to be a locally conformal almost Kähler manifold [4] if there is an open covering $\left\{U_{t}\right\}_{t \in I}$ of $M$ and a family $\left\{f_{t}\right\}_{t \in I}$ of $\mathbb{C}^{\infty}$-functions $f_{t}: U_{t} \longrightarrow \mathbb{R}$ such that, for any $t \in I$, the metric form

$$
g_{t}=\exp \left(-f_{t}\right) g_{\left.\right|_{U_{t}}}
$$

is an almost Kähler metric.

If the structures $\left(J, g_{t}\right)$ defined in (3) are Kähler, then $(M, J, g)$ is called locally conformal Kähler. Moreover, a locally conformal almost Kähler manifold $M$ is almost Kähler if and only if $d f_{t}=0$.

The Lee form is important because it characterizes locally conformal almost Kähler manifolds. Locally conformal almost Kähler manifolds were characterized by Vaisman in [4]. This is stated as follows: an almost Hermitian manifold $(M, J, g)$ is a locally conformal almost Kähler manifold if and only if there exists 1 -form $\omega$ such that

$$
\mathrm{d} \Omega=\omega \wedge \Omega, \mathrm{d} \omega=0 .
$$

Example 1. We consider the 4-dimensional manifold $M^{4}=\left\{p \in \mathbb{R}^{4} \mid x_{1} \neq 0, x_{2}>0\right\}$, where $p=\left(x_{1}, x_{2}, y_{1}, y_{2}\right)$ are the standard coordinates in $\mathbb{R}^{4}$. The vector fields,

$$
\begin{aligned}
& X_{i}=x_{2} \frac{\partial}{\partial x_{i}}, \\
& Y_{i}=\frac{1}{x_{2}^{3}} \frac{\partial}{\partial y_{i}}, \quad i=1,2,
\end{aligned}
$$

are linearly independent at each point of $M$. Let $g$ be the Riemannian metric on $M$ defined by $g\left(X_{i}, X_{j}\right)=g\left(Y_{i}\right.$, $\left.Y_{j}\right)=\delta_{i j}$, where $\delta_{i j}$ is the Kronecker symbol, $g\left(X_{i}, Y_{j}\right)=0$. That is, the form of the metric becomes

$$
g=\frac{1}{x_{2}^{2}}\left(\mathrm{~d} x_{1}^{2}+\mathrm{d} x_{2}^{2}\right)+x_{2}^{6}\left(\mathrm{~d} y_{1}^{2}+\mathrm{d} y_{2}^{2}\right)
$$

Let $J$ be the $(1,1)$-tensor field defined by $J X_{1}=Y_{1}$, $J X_{2}=-Y_{2}, J Y_{2}=X_{2}$, and $J Y_{1}=-X_{1}$. Thus, $(J, g)$ defines an almost Hermitian structure on $M^{4}$. The nonzero component of fundamental 2-form $J$ is

$$
\Omega\left(\frac{\partial}{\partial x_{1}}, \frac{\partial}{\partial y_{1}}\right)=-\frac{1}{x_{2}^{2}} \text { and } \Omega\left(\frac{\partial}{\partial x_{2}}, \frac{\partial}{\partial y_{2}}\right)=\frac{1}{x_{2}^{2}}
$$

and we have

$$
\Omega=\frac{1}{x_{2}^{2}}\left\{-\mathrm{d} x_{1} \wedge \mathrm{d} y_{1}+\mathrm{d} x_{2} \wedge \mathrm{d} y_{2}\right\} .
$$

Its differential gives

$$
\mathrm{d} \Omega=\frac{2}{x_{2}^{3}} \mathrm{~d} x_{1} \wedge \mathrm{d} y_{1} \wedge \mathrm{d} x_{2} .
$$

By letting

$$
\omega=-\frac{1}{x_{2}} \mathrm{~d} x_{2}
$$

we have

$$
\mathrm{d} \Omega=2 \omega \wedge \Omega .
$$

It is easy to see that $\mathrm{d} \omega=0$, and the dual vector field $B$ is given by

$$
B=-\frac{1}{x_{2}^{2}} X_{2} .
$$

Let us consider the open neighborhood $U$ of $M$ given by $U=\left\{p \in M^{4} \mid x_{2}>0\right\}$, and there exists a differentiable function $f$ on $U$ such that $\omega=\mathrm{d} f$, where $f=-\ln \left(x_{2}\right)$. By the aforementioned characterization given in $(4),\left(M^{4}, J, g\right)$ is a locally conformal almost Kähler manifold.

Next, we wish to study the relationship of the Levi-Civita connections induced by the locally conformal Kähler metrics $g_{t}$ and $g$.

Throughout this paper, $\Gamma(\Xi)$ will denote the $\mathscr{F}(M)$-module of differentiable sections of a vector bundle $\Xi$.

Let $\nabla$ and $\nabla^{t}$ be the Levi-Civita connections associated with the metrics $g$ and $g_{t}$, respectively. As is well known, they are connected by

$$
\nabla_{X}^{t} Y=\nabla_{X} Y-\frac{1}{2}\{\omega(X) Y+\omega(Y) X-g(X, Y) B\},
$$

for all $X, Y \in \Gamma(T M)$.

\section{Curvature Relations of Locally Conformal Almost Kähler Metrics}

Let $(M, J, g)$ be a $2 n$-dimensional almost Hermitian manifold. Here, we keep the formalism of local transformations and other formulas defined in the previous section. For the 
Riemann curvature $R$ of a metric $g$, we use the following convention:

$$
R(X, Y, Z, W)=g(R(X, Y) Z, W),
$$

where

$$
R(X, Y) Z=\nabla_{X} \nabla_{Y} Z-\nabla_{Y} \nabla_{X} Z-\nabla_{[X, Y]} Z,
$$

for any vector field $X, Y$, and $Z$ on $M$.

Let $\left\{E_{i}\right\}_{1 \leq i \leq 2 n}$ be the orthonormal basis with respect to g. The Ricci curvature tensor $\rho$ and the scalar curvature $\tau$ are given by

$$
\begin{aligned}
\rho(X, Y) & =\sum_{i=1}^{2 n} R\left(E_{i}, X, Y, E_{i}\right), \\
\tau & =\sum_{i=1}^{2 n} \rho\left(E_{i}, E_{i}\right) .
\end{aligned}
$$

Now, we consider the Ricci $*$-curvature tensor $\rho^{*}$ and the scalar $*$-curvature $\tau^{*}$ defined by

$$
\rho^{*}(X, Y)=\sum_{i=1}^{2 n} R\left(E_{i}, X, J Y, J E_{i}\right), \tau^{*}=\sum_{i=1}^{2 n} \rho^{*}\left(E_{i}, E_{i}\right) .
$$

Similarly, the curvatures corresponding to the metric $g_{t}$ will be denoted by $R^{t}, \rho^{t}, \tau^{t}, \rho^{t *}$, and $\tau^{t *}$, respectively.

Lemma 1. Let $(M, J, g)$ be a locally conformal almost Kähler manifold. Then, the curvature tensors $R^{t}$ and $R$ with respect to the metrics $g_{t}$ and $g$, respectively, are related as

$$
\begin{aligned}
R^{t}(X, Y) Z= & R(X, Y) Z+\frac{1}{2}\left\{\left(\nabla_{Y} \omega\right) Z+\frac{1}{2} \omega(Y) \omega(Z)\right\} X \\
& -\frac{1}{2}\left\{\left(\nabla_{X} \omega\right) Z+\frac{1}{2} \omega(X) \omega(Z)\right\} Y \\
& +\frac{1}{2} g(Y, Z)\left\{\nabla_{X} B+\frac{1}{2} \omega(X) B\right\} \\
& -\frac{1}{2} g(X, Z)\left\{\nabla_{Y} B+\frac{1}{2} \omega(Y) B\right\} \\
& -\frac{\|B\|^{2}}{4}\{g(Y, Z) X-g(X, Z) Y\},
\end{aligned}
$$

where $\|B\|^{2}=g(B, B)$.

Proof. Using the convention in (15) for the curvature tensors $R^{t}$ and $R$ and relation (13) and for any vector fields $X, Y$, and $Z$ tangent to $M$, the expressions

$$
\begin{aligned}
\nabla_{X}^{t} \nabla_{Y}^{t} Z= & \nabla_{X} \nabla_{Y} Z-\frac{1}{2} \omega(X) \nabla_{Y} Z-\frac{1}{2} \omega\left(\nabla_{Y} Z\right) \\
& +\frac{1}{2} g\left(X, \nabla_{Y} Z\right) B-\frac{1}{2} X(\omega(Y)) Z \\
& -\frac{1}{2} \omega(Y) \nabla_{X} Z+\frac{1}{4} \omega(X) \omega(Y) Z \\
& +\frac{1}{4} \omega(Y) \omega(Z) X-\frac{1}{4} \omega(Y) g(X, Z) B \\
& -\frac{1}{2} X(\omega(Z)) Y-\frac{1}{2} \omega(Z) \nabla_{X} Y \\
& +\frac{1}{4} \omega(X) \omega(Z) Y+\frac{1}{4} \omega(Y) \omega(Z) X \\
& -\frac{1}{4} \omega(Z) g(X, Y) B+\frac{1}{2} X(g(Y, Z)) B \\
& +\frac{1}{2} g(Y, Z) \nabla_{X} B-\frac{1}{4}\|B\|^{2} g(Y, Z) X .
\end{aligned}
$$

It is worth noting that

$$
\begin{aligned}
\nabla_{[X, Y]}^{t} Z= & \nabla_{[X, Y]} Z-\frac{1}{2} \omega([X, Y]) Z-\frac{1}{2} \omega(Z)[X, Y] \\
& +\frac{1}{2} g([X, Y], Z) B .
\end{aligned}
$$

Putting pieces (19) and (20) together, one obtains relation (18). This completes the proof.

Next, from the above lemma, we define $(0,2)$-tensor field $P$ by

$$
P(X, Y)=\left(\nabla_{X} \omega\right) Y+\frac{1}{2} \omega(X) \omega(Y)-\frac{1}{4}\|B\|^{2} g(X, Y),
$$

and this trace is given by

$$
\operatorname{trace} P=\operatorname{div} B-\frac{1}{2}(1-n)\|B\|^{2} .
$$

Lemma 2. The $(0,2)$-tensor field $P$ is symmetric.

Proof. For any vector fields $X$ and $Y$ tangent to $M$ and since $\omega$ is closed, we have 


$$
\begin{aligned}
P(X, Y)= & \left(\nabla_{Y} \omega\right) X+\frac{1}{2} \omega(X) \omega(Y)-\frac{1}{4}\|B\|^{2} g(X, Y) \\
= & Y(\omega(X))-\omega\left(\nabla_{Y} X\right)+\frac{1}{2} \omega(X) \omega(Y)-\frac{1}{4}\|B\|^{2} g(X, Y) \\
= & Y(\omega(X))-\omega([Y, X])-\omega\left(\nabla_{X} Y\right)+\frac{1}{2} \omega(X) \omega(Y) \\
& -\frac{1}{4}\|B\|^{2} g(X, Y) \\
= & \left(\nabla_{X} \omega\right) Y+\frac{1}{2} \omega(X) \omega(Y)-\frac{1}{4}\|B\|^{2} g(X, Y)
\end{aligned}
$$

which completes the proof.

The Lie derivative $g$ with respect to the vector field $B$ gives, for any vector fields $X$ and $Y$,

$$
\begin{aligned}
\left(L_{B} g\right)(X, Y) & =X(g(B, Y))-g([B, X], Y)-g(X,[B, Y]) \\
& =\left(\nabla_{X} \omega\right) Y+\left(\nabla_{Y} \omega\right) X=2\left(\nabla_{X} \omega\right) Y .
\end{aligned}
$$

The last equality of (24) follows from the fact that smooth 1 -form $\omega$ is closed.

Lemma 3. The dual vector field $B$ of $\omega$ preserves the metric $g$ if and only if the Lee form $\omega$ is $\nabla$-parallel.

The Riemannian curvatures are related by, for any $X, Y$, $Z$, and $W$ tangent to $M$,

$$
\begin{aligned}
\exp \left(f_{t}\right) R^{t}(X, Y, Z, W)= & R(X, Y, Z, W)+\frac{1}{2}\{g(X, W) P(Y, Z) \\
& -g(Y, W) P(X, Z)\}+\frac{1}{2}\{g(Y, Z) P(X, W) \\
& -g(X, Z) P(Y, W)\} .
\end{aligned}
$$

Let $\left\{E_{i}\right\}$ be the orthonormal basis with respect to $g$. Then, we have

$$
g\left(E_{i}, E_{j}\right)=\left\{\begin{array}{ll}
1 & \text { if } i=j, \\
0 & \text { if } i \neq j .
\end{array}\right\}
$$

Let $E_{i}^{t}=\exp \left(f_{t}\right)^{(1 / 2)} E_{i}$, for any $i=1,2, \ldots, 2 n$. Therefore, we have the following.

Lemma 4. The frame $\left\{E_{i}^{t}\right\}_{1 \leq i \leq 2 n}$ is the orthonormal basis with respect to $g_{t}$.

The following identities generalize the ones given in [9, p.216].

Lemma 5. The Ricci curvature tensors $\rho^{t}$ and $\rho$ with respect to $g_{t}$ and $g$, respectively, are related by

$$
\rho^{t}(X, Y)=\rho(X, Y)+(n-1) P(X, Y)+\frac{1}{2} g(X, Y) \operatorname{trace} P .
$$

Proof. Using Lemma 4 and for any vector fields $X$ and $Y$ tangent to $M$, one has

$$
\begin{aligned}
\rho^{t}(X, Y)= & \sum_{i=1}^{2 n} R^{t}\left(E_{i}^{t}, X, Y, E_{i}^{t}\right)=\sum_{i=1}^{2 n} \exp \left(f_{t}\right) R^{t}\left(E_{i}, X, Y, E_{i}\right) \\
= & \sum_{i=1}^{2 n} R\left(E_{i}, X, Y, E_{i}\right)+\frac{1}{2}\left\{\sum_{i=1}^{2 n} g\left(E_{i}, E_{i}\right) P(X, Y)\right. \\
& \left.-\sum_{i=1}^{2 n} g\left(X, E_{i}\right) P\left(E_{i}, Y\right)\right\} \\
+ & \frac{1}{2}\left\{\sum_{i=1}^{2 n} g(X, Y) P\left(E_{i}, E_{i}\right)-\sum_{i=1}^{2 n} g\left(E_{i}, Y\right) P\left(X, E_{i}\right)\right\} \\
= & \rho(X, Y)+(n-1) P(X, Y)+\frac{1}{2} g(X, Y) \operatorname{trace} P,
\end{aligned}
$$

which completes the proof.

Also, corresponding Ricci *-curvatures are related by

$$
\rho^{t *}(X, Y)=\rho^{*}(X, Y)+\frac{1}{2}\{P(X, Y)+P(J X, J Y)\} .
$$

Corollary 1. The scalar curvatures $\tau^{t}$ and $\tau$ are related by

$$
\exp \left(-f_{t}\right) \tau_{t}=\tau+(2 n-1)\left\{\operatorname{div} B-\frac{1}{2}(1-n)\|B\|^{2}\right\} \text {. }
$$

Proof. Using Lemma 4 and the scalar curvature $\tau^{t}$, we have

$$
\tau^{t}=\sum_{i=1}^{2 n} \rho^{t}\left(E_{i}^{t}, E_{i}^{t}\right)=\exp \left(f_{t}\right) \sum_{i=1}^{2 n} \rho^{t}\left(E_{i}, E_{i}\right) .
$$

Then, applying equation (26) to (31), we get

$$
\begin{aligned}
\exp \left(-f_{t}\right) \tau^{t} & =\sum_{i=1}^{2 n} \rho\left(E_{i}, E_{i}\right)+(n-1) \sum_{i=1}^{2 n} P\left(E_{i}, E_{i}\right)+n \text { trace } P \\
& =\tau+(2 n-1) \operatorname{trace} P \\
& =\tau+(2 n-1)\left\{\operatorname{div} B-\frac{1}{2}(1-n)\|B\|^{2}\right\} .
\end{aligned}
$$

Therefore,

$$
\exp \left(-f_{t}\right) \tau^{t}=\tau+(2 n-1)\left\{\operatorname{div} B-\frac{1}{2}(1-n)\|B\|^{2}\right\}
$$

which completes the proof.

Now, if we consider a relation between scalar *-curvature $\tau^{t *}$ and $\tau^{*}$, we get the following.

Corollary 2. The scalar $*$-curvatures $\tau^{t *}$ and $\tau^{*}$ are related by

$$
\exp \left(-f_{t}\right) \tau^{t *}=\tau^{*}+\operatorname{div} B+(n-1)\|B\|^{2}
$$


Proof. The scalar *-curvature $\tau^{t *}$ is given by

$$
\tau^{t^{*}}=\sum_{i=1}^{2 n} \rho^{t *}\left(E_{i}^{t}, E_{i}^{t}\right)=\exp \left(f_{t}\right) \sum_{i=1}^{2 n} \rho^{t *}\left(E_{i}, E_{i}\right) .
$$

Now, applying relation (29) to (35), we compute

$$
\begin{aligned}
\exp \left(-f_{t}\right) \tau^{t *} & =\sum_{i=1}^{2 n} \rho^{t^{*}}\left(E_{i}, E_{i}\right) \\
& =\sum_{i=1}^{2 n} \rho^{*}(X, Y)+\frac{1}{2} \sum_{i=1}^{2 n}\left\{P\left(E_{i}, E_{i}\right)+P\left(J E_{i}, j E_{i}\right)\right\} \\
& =\tau^{*}+\operatorname{div} B+(n-1)\|B\|^{2}
\end{aligned}
$$

Hence,

$$
\exp \left(-f_{t}\right) \tau^{t^{*}}=\tau^{*}+\operatorname{div} B+(n-1)\|B\|^{2},
$$

as required.

Gray in [7] considered some curvature identities for Hermitian and almost Hermitian manifolds. Let $\mathscr{L}$ be the class of almost Hermitian manifolds as defined in [7]. Then, the manifold under consideration is an element of class $\mathscr{L}$. Now, consider as in [7] the curvature operator $R^{t}$ of a locally conformal almost Kähler manifold $M$ :

$$
\begin{aligned}
(1) R^{t}(X, Y, Z, W)= & R^{t}(X, Y, J Z, J W), \\
(2) R^{t}(X, Y, Z, W)-R^{t}(J X, J Y, Z, W)= & R^{t}(J X, Y, J Z, W), \\
& +R^{t}(J X, Y, Z, J W), \\
(3) R^{t}(X, Y, Z, W)= & R^{t}(J X, J Y, J Z, J W),
\end{aligned}
$$

for any $X, Y, Z$, and $W$ tangent to $M$. Item (1) is called a Kähler identity if $M$ is a locally conformal Kähler manifold (see [7] for more details and references therein).

We will focus, throughout the rest of this note, on item (1). Using further notations as in [7], we denoted by $\mathscr{L}_{i}$ the subclass of manifolds whose curvature operator $R^{t}$ satisfies identity (i). Here, (i) may be either item (1), (2), or (3) above. As in [7], it is easy to see that

$$
\mathscr{L}_{1} \subseteq \mathscr{L}_{2} \subseteq \mathscr{L}_{3} \subseteq \mathscr{L}
$$

Therefore, we have the following result.

Lemma 6. If a locally conformal almost Kähler manifold is in class $\mathscr{L}_{1}$, then the equality holds

$$
\tau^{*}-\tau=2(n-1) \operatorname{trace} P .
$$

Proof. The proof follows from a straightforward calculation using the fact that, for any vector fields $X$ and $Y$ tangent to $M$, we have

$$
\begin{aligned}
\rho^{t}(X, Y) & =\sum_{i=1}^{2 n} \exp \left(f_{t}\right) R^{t}\left(E_{i}, X, Y, E_{i}\right) \\
& =\sum_{i=1}^{2 n} \exp \left(f_{t}\right) R^{t}\left(E_{i}, X, J Y, J E_{i}\right)=\rho^{* t}(X, Y)
\end{aligned}
$$

which leads to

$$
\begin{aligned}
\left(\rho^{*}-\rho\right)(X, Y)= & \left(n-\frac{3}{2}\right) P(X, Y)+\frac{1}{2} g(X, Y) \operatorname{trace} P \\
& -\frac{1}{2} P(J X, J Y) .
\end{aligned}
$$

This completes the proof.

Relation (41) leads to

$$
\begin{aligned}
\tau^{t} & =\sum_{i=1}^{2 n} \sum_{j=1}^{2 n} R^{t}\left(E_{j}, E_{i}, E_{i}, E_{j}\right) \\
& =\sum_{i=1}^{2 n} \sum_{j=1}^{2 n} R^{t}\left(E_{j}, E_{i}, J E_{i}, J E_{j}\right)=\tau^{t *} .
\end{aligned}
$$

Theorem 1. Let $(M, J, g)$ be a 2n-dimensional compact locally conformal almost Kähler manifold with $n>1$ and contained in $\mathscr{L}_{1}$. If

$$
\tau^{*}=\tau
$$

then $(M, J, g)$ is an almost Kähler manifold.

Proof. By Lemma 6, we have $\tau^{*}-\tau=2(n-1) \operatorname{trace} P$, with trace $P=\operatorname{div} B-(1 / 2)(1-n)\|B\|^{2}$. Taking into account this, integrating relation (40), and using Green's theorem, we have $0=\int_{M}\left\{\tau^{*}-\tau\right\}=(n-1)^{2} \int_{M}\|B\|^{2}$. Hence, under our assumption, we obtain $B=0$. Therefore, $\omega=0$ identically on $M$. Hence, $(M . J, g)$ is an almost Kähler manifold.

As an example for this theorem, we have compact flat locally almost Kähler manifolds. Compact flat manifolds have been detailed in [8] and references therein.

\section{Lee Form and Canonical Foliations}

Let $(M, J, g)$ be a locally conformal almost Kähler manifold, and assume that the Lee form $\omega$ is never vanishing on $M$. Then, $\omega=0$ defines on $M$ an integrable distribution, and hence a foliation $\mathscr{F}$, on $M$ (see [9] for more details and references therein).

Let $D:=\operatorname{ker} \omega$ be the distribution on $M$ and $D^{\perp}$ be the distribution spanned by the vector field $B$. Then, we have the following decomposition:

$$
T M=D \oplus D^{\perp}
$$

where $\oplus$ denotes the orthogonal direct sum. By decomposition (45), any $X \in \Gamma(T M)$ is written as 


$$
X=Q X+Q^{\perp} X
$$

where $Q$ and $Q^{\perp}$ are the projection morphisms of $T M$ into $D$ and $D^{\perp}$, respectively. Here, it is easy to see that $Q^{\perp} X=\left(1 /\|B\|^{2}\right) \omega(X) B$, and

$$
X=Q X+\frac{1}{\|B\|^{2}} \omega(X) B .
$$

Let $\mathscr{F}$ be a foliation on a locally conformal almost Kähler manifold $(M, J, g)$ of codimension 1 . The metric $g$ is said to be bundle-like for the foliation $\mathscr{F}$ if the induced metric on the transversal distribution $D^{\perp}$ is parallel with respect to the intrinsic connection on $D^{\perp}$. This is true if and only if the Levi-Civita connection $\nabla$ of $(M, J, g)$ satisfies (see $[10,11]$ for more details)

$$
g\left(\nabla_{Q^{\perp} Y} Q X, Q^{\perp} Z\right)+g\left(\nabla_{Q^{\perp} Z} Q X, Q^{\perp} Y\right)=0,
$$

for any $X, Y, Z \in \Gamma(T M)$. A foliation $\mathscr{F}$ is said to be Riemannian on $(M, J, g)$ if the Riemannian metric $g$ on $M$ is bundle-like for $\mathscr{F}$.

Let $\mathscr{F}^{\perp}$ be the orthogonal complementary foliation generated by $B$. Now, we provide necessary and sufficient conditions for the metric on an locally conformal almost Kähler manifold to be bundle-like for foliations $\mathscr{F}$ and $\mathscr{F}^{\perp}$.

Theorem 2. Let $(M, J, g)$ be a locally conformal almost Kähler manifold, and let $\mathscr{F}$ be a foliation on $M$ of codimension 1. Then, the following assertions are equivalent:

(i) The foliation $\mathscr{F}$ is Riemannian.

(ii) The Lee vector field $B$ is autoparallel with respect to $\nabla$, that is,

$$
\nabla_{B} B=B(\ln (\|B\|)) B
$$

Proof. For any $X, \quad Y, \quad Z \in \Gamma(T M)$, we have $Q^{\perp} Y=\left(1 /\|B\|^{2}\right) \omega(Y) B$ and $Q^{\perp} Z=\left(1 /\|B\|^{2}\right) \omega(Z) B$, and the left-hand side of (48) gives

$$
g\left(\nabla_{Q^{\perp} Y} Q X, Q^{\perp} Z\right)+g\left(\nabla_{Q^{\perp} Z} Q X, Q^{\perp} Y\right)=\frac{2}{\|B\|^{2}} \omega(Y) \omega(Z) \omega\left(\nabla_{B} Q X\right),
$$

for which the equivalence follows.

Let $M^{\prime}$ be a leaf of the distribution $D$. Since $M^{\prime}$ is a submanifold of $M$ and for any $X, Y \in \Gamma\left(T M^{\prime}\right)$, we have

$$
\begin{aligned}
& \nabla_{X} Y=\nabla_{X}^{\prime} Y+\alpha(X, Y), \\
& \nabla_{X} B=-A_{B} X+\nabla_{X}^{\prime} B,
\end{aligned}
$$

where $\nabla^{\prime}$ and $\alpha$ are the Levi-Civita connection and the second fundamental form of $M^{\prime}$, respectively. Here, $A_{B}$ is the shape operator with respect to $B$. On the contrary, we have $g\left(\nabla_{X} B, B\right)=X(\omega(B))-g\left(\nabla_{X} B, B\right)$; hence,

$$
g\left(\nabla_{X}^{\prime}{ }^{\prime} B, B\right)=\frac{1}{2} X(\omega(B)),
$$

for any $X \in \Gamma\left(T M^{\prime}\right)$. Therefore, Weingarten formula (52) becomes

$$
\nabla_{X} B=-A_{B} X+\frac{1}{2} X(\omega(B)) B
$$

Proposition 1. Let $(M, J, g)$ be a locally conformal almost Kähler manifold. Then, the mean curvature vector field $H^{\prime}$ of the leaves of the integrable distribution $D$ defined in (45) is given by

$$
H^{\prime}=\frac{1}{2 n-1}\left(\operatorname{div}_{\left.\right|_{M^{\prime}}} B\right) B .
$$

Moreover, these leaves are totally geodesic hypersurfaces of $M$ if and only if the dual vector field $B$ of $\omega$ preserves their metrics.

Proof. Let $M^{\prime}$ be a leaf of the integrable distribution $D$. Using (51) and (54), the second fundamental form of $M^{\prime}$ gives

$$
\alpha(X, Y)=g\left(A_{B} X, Y\right) B=g\left(\nabla_{X} B, Y\right) B,
$$

for any $X, Y \in \Gamma\left(T M^{\prime}\right)$. Fixing a local orthonormal frame $\left\{e_{1}, \ldots, e_{2 n-1}\right\}$ in $T M^{\prime}$, one has

$$
H=\frac{1}{2 n-1} \sum_{i=1}^{2 n-1} \alpha\left(e_{i}, e_{i}\right)=\frac{1}{2 n-1}\left(\operatorname{div}_{\left.\right|_{M^{\prime}}} B\right) B .
$$

The last assertion follows, and this completes the proof. Therefore, we have the following result.

Corollary 4. Let $(M, J, g)$ be a locally conformal almost Kähler manifold. Then, the leaves $M^{\prime}$ of the distribution $D$ in (45) are minimal if and only if the dual vector field $B$ is incompressible along $M^{\prime}$.

\section{Data Availability}

No data were used to support this study.

\section{Conflicts of Interest}

The authors declare that they have no conflicts of interest regarding the publication of this paper.

\section{Acknowledgments}

This work was based on the research supported wholly/in part by the National Research Foundation of South Africa (Grant nos. 95931 and 106072).

\section{References}

[1] S. Dragomir and L. Ornea, "Locally conformal kähler geometry," 2012.

[2] M. P. Libermann, Sur les Automorphismes Infinitesimaux des Structures Symplectiques et des Structures de Contact, (French) 
1959 Colloque Géom, Difference Globale, Brussels, Belgium, 1958.

[3] A. Gray, "Some examples of almost hermitian manifolds," Illinois Journal of Mathematics, vol. 10, no. 2, pp. 353-366, 1966.

[4] I. Vaisman, "On locally conformal almost kähler manifolds," Israel Journal of Mathematics, vol. 24, no. 3, pp. 338-351, 1976.

[5] Z. Olszak, "On the existence of generalized complex space forms," Israel Journal of Mathematics, vol. 65, no. 2, pp. 214-218, 1989.

[6] D. Catalano, F. Defever, R. Deszcz, M. Hotloś, and Z. Olszak, "A note on almost kähler manifolds," Abhandlungen aus dem Mathematischen Seminar der Universität Hamburg, vol. 69, no. 1, pp. 59-65, 1999.

[7] A. Gray, "Curvature identities for hermitian and almost hermitian manifolds," Tohoku Mathematical Journal, vol. 4, pp. 601-612, 1976.

[8] L. S. Charlap, "Compact flat riemannian manifolds I," Annals of Mathematics, vol. 2, pp. 15-30, 1965.

[9] T.-W. Kim and H.-K. Pak, "A subfoliation of a Cr-foliation on a locally conformal almost kahler manifold," Journal of the Korean Mathematical Society, vol. 41, no. 5, pp. 865-874, 2004.

[10] A. Bejancu and H. R. Farran, Foliations and Geometric Structures, Mathematics and its Applications, Springer, Berlin, Germany, 2006.

[11] P. Tondeur, Geometry of Foliations, Monographs in Mathematics, Birkhäuser, Basel, Switzerland, 1997. 